\title{
DUCTAL CARCINOMA IN SITU OF THE BREAST: FIRST ANALYSIS OF 167 CASES
}

André Mattar ${ }^{1}$, Marcellus do Nascimento Moreira Ramos'1, Andressa Gonçalves Amorim¹, Jorge Yoshinori Shida', Roberto Hegg', Luiz Henrique Gebrim¹

${ }^{1}$ Hospital Pérola Byington - São Paulo (SP), Brazil.

Objectives: Ductal carcinoma in situ (DCIS) of the breast represents a heterogeneous group of neoplastic lesions confined to the ducts and lobules of the breast, which differ in histological grade and biological potential. As the principal finding is microcalcifications, the presence of DCIS is more frequent in populations that are submitted to mammography screening. This condition is usually treated with local surgery and adjuvant radiotherapy. The use of sentinel node biopsy is reserved for large tumors when the risk of invasion is higher and in radical surgeries. Methodology: We have performed a retrospective analysis of 157 cases of DCIS diagnosed by percutaneous biopsy treated in our institution between 2011 and 2013. All the patients had an initial biopsy and had a definitive surgery in our hospital. We have initially reviewed the age, grade, the presence of necrosis, type of surgery, and the presence of axillary staging by sentinel node biopsy (SNB) or axillary sampling (AS). Results: From 167 cases we saw 18 cases that initially were diagnosed as DCIS, but were confirmed to be invasive after surgery. Most of our population were more than 50 years old (64\%). Interestingly $30 \%$ of our patients were between 40-49 years, a range that is not contemplated in the program of mammographic screening determined by the National Cancer Institute (INCA). Most of our patients were treated with partial mastectomy (70\%) and radical surgery in $30 \%$. In $9 \%$ of the cases, a AS was performed and, in $20 \%$, SNB was performed. There was no lymph node positive in our analyses. Conclusion: DCIS is an important disease and our data showed that the prevalence in the range age 40-49 is high and the INCA screening programme should be reviewed. The use of axillary staging should be discouraged for DCIS. 